\title{
Gravitational perturbations as a source of timing noise
}

\author{
A. E. Rodin
}

PRAO ASC LPI, Pushchino, Moscow region, 142292, Russia

\begin{abstract}
In assumption that pulsar gravitationally interacts with its surroundings the relationships between the pulsar rotational and orbital parameters are derived and the solutions for PSR B1620-26 and PSR B1822-09 are presented.
\end{abstract}

Many pulsars show timing activity which is expressed in presence of the high order time derivatives of the rotational frequency. Presence of this derivatives can be explained by direct gravitational interactions between a pulsar and a perturbing body (Joshi \& Rasio 1997; Rodin 1997; Thorsett et al. 1999). A method have been developed which allows to derive orbital elements of a pulsar on the basis of its rotational parameters.

Table 1 shows the relationship between rotational frequency derivatives and orbital parameters of a pulsar. On the basis of the formulas from the Table 1 and the rotational parameters of PSR B1620-26 and PSR B1822-09 one can derive orbital elements. The orbital elements in assuming that $e=0$ for PSR B1620-26 are almost the same comparing with that from the paper (Thorsett et al. 1999). For PSR 1822-09 there are two possible solutions: 1) hyperbolic, eccentricity $e=3.15$, semimajor axis $a=2.2$ a. u., mass of perturbing body $m_{2} \sin i=$ $\left.3.7 \cdot 10^{-4} M_{\odot} ; 2\right)$ elliptic, $e=0.27, a=3.6$ a. u., $m_{2} \sin i=1.2 \cdot 10^{-4} M_{\odot}$.

Fig. 1 shows theoretical curves of $\Delta \varphi / \nu, \Delta \nu / \nu, \Delta \dot{\nu} / \nu, \ddot{\nu} / \nu, \nu^{(3)} / \nu, \nu^{(4)} / \nu$ along with the observational data of PSR B1822-09 (Shabanova, 1998). There is satisfactory coincidence of the theoretical curves and the observational data. The gravitational perturbation approach proposes the better explanation of the rotational activity of PSR B1822-09 comparing with the glitch theory (Shabanova, 1998) and gives reliable estimates of mass of and distance to a perturbing body for both PSR B1620-26 and PSR B1822-09.

Deviations of the observational data from the theoretical curves still remain and can be explained by proper pulsar noise caused, for example, by a pulsar inner event induced by the tidal wave from the close passage of a massive body.

\section{References}

Joshi, K. J., \& Rasio, F. A. 1997, ApJ, 479, 948.

Rodin, A. E. 1997, In book: "Problems of modern radio astronomy" (S.- Peterburg), 2, 193 (in Russian).

Shabanova, T. V. 1998, A\&A, 337, 723.

Thorsett, S. E., Arzoumanian, Z., Camilo, F. M., \& Lyne, A. G. 1999, ApJ, 523, 763. 
Table 1. The relationship between pulsar rotational frequency derivatives and orbital parameters of the pulsar perturbed by a massive body on the hyperbolic $(e>1)$, parabolic $(e=1)$ and elliptic orbit $(e<1)$. Denominations: $\alpha=x \sin \omega, \beta=x \sqrt{e^{2}-1} \cos \omega$ (hyperbolic orbit), $\beta=x \sqrt{1-e^{2}} \cos \omega$ (elliptic orbit), $\beta=x \cos \omega$ (parabolic orbit) $n$ - mean motion, $e$ - eccentricity, $x$ - projection of semimajor axis (parameter of orbit in the case of parabola) on the line of sight, $\omega$ - longitude of pericenter, $\nu, \dot{\nu}$ - pulsar rotational frequency and its derivative as if no perturbations. Assumptions were made: 1) expansion of pulsar phase was performed about the known time of pericenter passage, 2) proper 2 nd derivative of pulsar rotational frequency $\ddot{\nu}=0$.

\begin{tabular}{|c|cccccc|}
\hline & $f / \nu$ & $f / \nu$ & $f / \nu$ & $f^{(3)} / \nu$ & $f^{(4)} / \nu$ & $f^{(5) / \nu}$ \\
\hline$e>1$ & $1+\frac{\beta n}{(e-1)}$ & $-\frac{\alpha n^{2}}{(e-1)^{2}}+\frac{i}{\nu}$ & $-\frac{\beta n^{3}}{(e-1)^{4}}$ & $\frac{(1+3 e) \alpha n^{4}}{(e-1)^{5}}$ & $\frac{(1+9 e) \beta n^{5}}{(e-1)^{7}}$ & $-\frac{\left(1+24 e+45 e^{2}\right) \alpha n^{6}}{(e-1)^{8}}$ \\
$e=1$ & $1+\beta n$ & $-\alpha n^{2}+\frac{\dot{\nu}}{\nu}$ & $-2 \beta n^{3}$ & $16 \alpha n^{4}$ & $40 \beta n^{5}$ & $-560 \alpha n^{6}$ \\
$e<1$ & $1-\frac{\beta n}{(1-e)}$ & $-\frac{\alpha n^{2}}{(1-e)^{2}}+\frac{\dot{\nu}}{\nu}$ & $\frac{\beta n^{3}}{(1-e)^{4}}$ & $\frac{(1+3 e) \alpha n^{4}}{(1-e)^{5}}$ & $-\frac{(1+0 e) \beta n^{5}}{(1-e)^{7}}$ & $-\frac{\left(1+2 d e+45 e^{2}\right) \alpha n^{6}}{(1-e)^{8}}$ \\
\hline
\end{tabular}
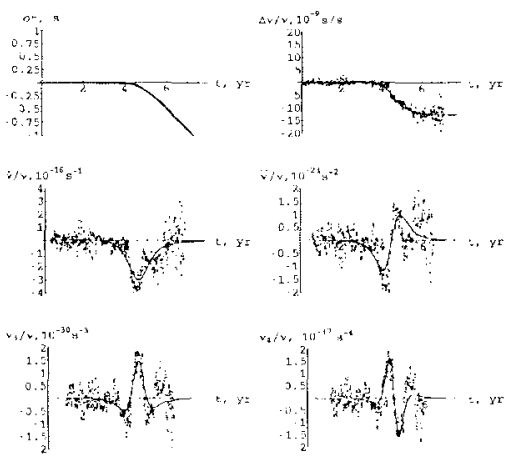
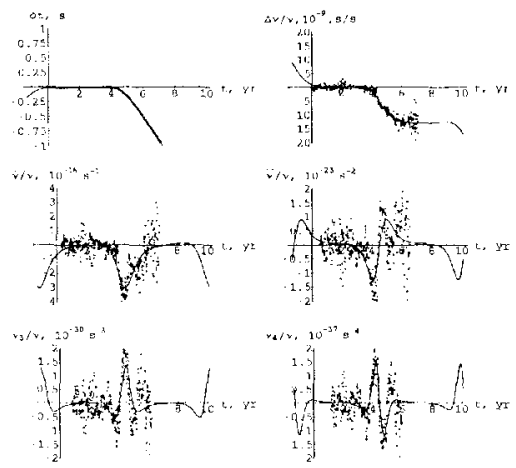

Figure 1. Variations of the pulsar rotational phase, frequency and its derivatives of PSR B1822-09 in the hyperbolic (1st and 2nd column) and elliptic case ( $3 \mathrm{rd}$ and 4 th column). Points are observational data, line is theoretical curve. Variations of the frequency and its derivatives were calculated as finite difference of the smoothed data. 